ARTICLE

\title{
Towards explicit regulating-ion-transport: nanochannels with only function-elements at outer-surface
}

Qun Ma1, Yu Li ${ }^{1}$, Rongsheng Wang ${ }^{1}$, Hongquan $\mathrm{Xu}^{1}$, Qiujiao $\mathrm{Du}^{2}$, Pengcheng Gao ${ }^{1 凶} \&$ Fan Xia (i) ${ }^{1 凶}$

Function elements (FE) are vital components of nanochannel-systems for artificially regulating ion transport. Conventionally, the $\mathrm{FE}$ at inner wall ( $\mathrm{FE}_{\mathrm{IW}}$ ) of nanochannel- ${ }^{-}$systems are of concern owing to their recognized effect on the compression of ionic passageways. However, their properties are inexplicit or generally presumed from the properties of the FE at outer surface $\left(\mathrm{FE}_{\mathrm{OS}}\right)$, which will bring potential errors. Here, we show that the $\mathrm{FE}_{\mathrm{OS}}$ independently regulate ion transport in a nanochannel- system without $\mathrm{FE}_{\mid \mathrm{W}}$. The numerical simulations, assigned the measured parameters of $\mathrm{FE}_{\mathrm{OS}}$ to the Poisson and Nernst-Planck (PNP) equations, are well fitted with the experiments, indicating the generally explicit regulating-ion-transport accomplished by $\mathrm{FE}_{\mathrm{OS}}$ without $\mathrm{FE}_{\mathrm{IW}}$. Meanwhile, the $\mathrm{FE}_{\mathrm{OS}}$ fulfill the key features of the pervious nanochannel systems on regulating-ion-transport in osmotic energy conversion devices and biosensors, and show advantages to (1) promote power density through concentrating FE at outer surface, bringing increase of ionic selectivity but no obvious change in internal resistance; (2) accommodate probes or targets with size beyond the diameter of nanochannels. Nanochannel-systems with only FEos of explicit properties provide a quantitative platform for studying substrate transport phenomena through nanoconfined space, including nanopores, nanochannels, nanopipettes, porous membranes and two-dimensional channels.

\footnotetext{
${ }^{1}$ State Key Laboratory of Biogeology and Environmental Geology, Engineering Research Center of Nano-Geomaterials of Ministry of Education, Faculty of Materials Science and Chemistry, China University of Geosciences, Wuhan, P. R. China. ${ }^{2}$ School of Mathematics and Physics, China University of Geosciences, Wuhan, P. R. China.凶email: pcgao@cug.edu.cn; xiafan@cug.edu.cn
} 
$\mathrm{N}$ anochannel-systems are artificial passages of ions and molecules with unique controllable performances ${ }^{1,2}$. They have been widely used in sensing ${ }^{3-5}$, drug release ${ }^{6,7}$, separation $^{8-11}$, nanofluidic ${ }^{12}$, nanoelectrochemistry ${ }^{13,14}$, and energy conversion ${ }^{15-17}$, owing to their adjustable geometries at the nanoscale, versatile chemical compositions, and strong mechanical strength. The nanochannel-systems usually consist of three components: (1) nanochannels, (2) function elements at outer surface $\left(\mathrm{FE}_{\mathrm{OS}}\right)$, and (3) function elements at inner wall $\left(\mathrm{FE}_{\mathrm{IW}}\right)^{18,19}$. However, in traditional nanochannel-systems, there are two troublesome "black boxes" which are not well addressed: one is the role of $\mathrm{FE}_{\mathrm{OS}}$ on ion transport, which has been longtermed neglected; and the other is inexplicit chemical and physical properties of FE located deep inside nanochannel which is subject to that few test techniques with test tips or testing liquids, that can sufficiently contact with FE in the confined space at the nanoscale ${ }^{2}$.

Currently, both theoretical ${ }^{20,21}$ and experimental investigation $^{22-25}$ showed the synergistic effects of $\mathrm{FE}_{\mathrm{OS}}$ on regulatingion-transport in the presence of $\mathrm{FE}_{\mathrm{IW}}$. Compared with the confined space in nanochannel, relatively more free-spaces of OS endow $\mathrm{FE}_{\mathrm{OS}}$ with advanced characteristics, such as easy to immobilize, available for precise characterizations, receptive for foreign substrates, and potential application in new scenarios. However, till now the properties of most FE are still inexplicit or generally presumed from the measurable $\mathrm{FE}_{\mathrm{OS}}{ }^{26,27}$, which would bring potential errors.

Here, we confined FE at the outer surface (OS) and the edge of IW with a minimum depth as $7.5 \mathrm{~nm}(\sim 0.01 \%$ of total IW $)$ in nanochannels through the threshold effect of reducing-diameter down to $11 \pm 3 \mathrm{~nm}$, which is detectable for a host of techniques and termed as $\mathrm{FE}_{\mathrm{OS}}$. The $\mathrm{FE}_{\mathrm{OS}}$ have been certified to regulate ion transport independently and their mechanism can be well demonstrated through the Poisson and Nernst-Planck equations assigned by measured properties from atomic force microscope (AFM), time of flight secondary ion mass spectrometry (ToFSIMS), and solid-surface zeta potential analyzer (SSZPA). The $\mathrm{FE}_{\mathrm{OS}}$ fulfill the key capabilities of nanochannels in osmotic energy conversion and biosensing and bring new features: (1) increase of ionic selectivity but no obvious change in resistance and (2) accommodating probes or targets with size beyond the diameter of nanochannels.

\section{Results}

Designed nanochannel-systems. Different from previous nanochannel-systems (Fig. 1a, b), we designed a new nanochannel-system (Fig. 1c). In the 1st stage in Fig. 1a and 2nd stage in Fig. 1b, both the physicochemical properties and function on regulating-ion-transport of nanochannel system are partially unclear due to the limitation for the characterization of $\mathrm{FE}_{\mathrm{IW}}$. While, using the nanochannel-system only consisting of independent $\mathrm{FE}_{\mathrm{OS}}$ (Fig. 1c) will avoid addressing the two problems: one is the role of $\mathrm{FE}_{\mathrm{OS}}$ on regulating-ion-transport and the other is unclear physicochemical properties of $\mathrm{FE}_{\mathrm{IW}}$. Hence, the explicit relationship between the physicochemical properties of FE and function of nanochannel-systems could be realized.

Fabrications of nanochannel-system with only $\mathrm{FE}_{\mathrm{OS}}$. We built a nanochannel-system using an anodic aluminum oxide (AAO) membrane deposited by $\mathrm{Au}$ at the one side as nanochannels (named as none@OS) (Fig. 2a-e, Fig. S1) 22,23 . For the present nanochannels, their surfaces could be divided into two parts: (1) OS refers to the outermost surface of $\mathrm{Au}$ and $\mathrm{AAO}$ at the opposite side. Because FE didn't attach to the outermost layer of AAO, the OS only refers to the outermost layer of $\mathrm{Au}$ in the present work
(Fig. S2). (2) Inner wall (IW) refers to the residual surface of the nanochannels except for the OS (Fig. 2a). We reduced the diameter of nanochannels from $25 \pm 5 \mathrm{~nm}$ to $11 \pm 3 \mathrm{~nm}$ by prolonging deposition time $(0.1 \mathrm{~nm} / \mathrm{s}$ for $2000 \mathrm{~s})$ on purpose of restraining FE from entering the IW of nanochannels through the threshold effect (meaning that the stacking FE initially at the opening of nanochannels excluded the subsequent FE from entering IW to a great extent) (Fig. 2d, e and S3-S6), differing from the relatively free diffusion in nanochannel within larger channel diameter $23,26-29$. Then, the three different FE, including polyacrylic acid (PAA, Mw 5000) through Van der Waals' force, poly(ethylene imine) (PEI, Mw 10,000) through Van der Waals' force ${ }^{30}$ and DNA (Mw 11,000) through Au-thiol interactions ${ }^{22,23}$ respectively, were attached to the OS of none@OS (Figs. S7 and S8). The as-obtained nanochannel-systems were named as PAA@OS,PEI@OS, and DNA@OS, respectively.

Definitions of $\mathrm{FE}_{\mathrm{OS}}$ in nanochannel systems. We further defined $\mathrm{FE}_{\mathrm{OS}}$ in the present nanochannel-system. Frankly, limited by current technologies, we cannot attach FE purely at the OS but not at the IW (Fig. 2f). Therefore, the depth of FE at the IW of the nanochannels was measured to be as much as $180 \mathrm{~nm}$ (PAA@OS), 75 nm (PEI@OS), and 23 nm (DNA@OS), using the ToF-SIMS and the scanning electron microscope (SEM) ${ }^{23}$, which occupied a small part of the total depth of IW $(65 \mu \mathrm{m})$ as $0.28 \%$, $0.12 \%$, and $0.04 \%$, respectively. (Fig. $2 \mathrm{~g}$ and S9). Compared to the distribution percentage of $\mathrm{FE}$ in the 1st stage through random indraft $(\approx 100 \%)^{26,27,29}$ and in the 2nd stage through Au-S interaction $(5-30 \%)^{23}$, the depth of FE occupied a tiny percentage $(<0.3 \%)$ of the total depth of IW in this work, which could be attributed to the threshold effect during the penetration process of FE. We found that the FE depth at IW decreased with their molar mass that manifested the threshold effect (right figure in Fig. 2g, iv). We, therefore, defined the $\mathrm{FE}_{\mathrm{OS}}$ in this work (Fig. 2f), consisting of the FE at the OS of Au side and the FE at the IW existing near the opening of the nanochannels (Fig. $2 \mathrm{~g}$ and S10).

Explicit role of $\mathrm{FE}_{\mathrm{O}}$ in regulating-ion-transport. We then investigated the role of $\mathrm{FE}_{\mathrm{OS}}$ in the regulating-ion-transport in the nanochannel-system using a two-electrode cell with two symmetric $\mathrm{Ag} / \mathrm{AgCl}$ electrodes and $0.1 \mathrm{M} \mathrm{KCl}$ solution (Fig. 3a). The current-voltage (I-V) plots of none@OS is asymmetric (Fig. 3b) owing to the asymmetric structure and surface properties (Figs. S5 and S6), classified as ion-current rectification (ICR) behavior, in which $I_{+2 \mathrm{~V}}$ (current at $+2 \mathrm{~V}$ ) / $I_{-2 \mathrm{~V}}$ (current at $-2 \mathrm{~V})$ is defined as ICR ratio $\left(f_{\mathrm{rec}}\right)^{31}$. After attached $\mathrm{FE}_{\mathrm{OS}}$, the $f_{\text {rec }}$ increased (for PAA@OS and DNA@OS) and the $f_{\text {rec }}$ decrease lower than 1 with an opposite polarity (for PEI@OS) (Fig. 3f). The variation of ion current above shows a similar trend as the previous reports (FE with distributions in the 1st stage described in Fig. 1 $)^{30}$. This effect was ascribed to the enhancement of negative charge at outer surface from highly negatived PAA rich in hydroxyl in PAA@OS or DNA with phosphodiester skeleton in DNA@OS (Fig. S11), or charge reversal of surface charge for the highly positive PEI@OS rich in amino, leading to the enhancement of ion accumulation and depletion (Fig. S16). In addition, the ion transport of nanochannels with independent $\mathrm{FE}_{\mathrm{OS}}$ was affected by ion strength (Fig. S13). Therefore, we estimated that the $\mathrm{FE}_{\mathrm{OS}}$ influenced the ion currents through nanochannelsystem above independently.

In the 1 st and 2 nd stage, the numerical simulations coupled the Poisson-Nernst-Planck (PNP) equations ${ }^{32}$ among the classical equations with steady state continuity equations were performed for $\mathrm{FE}_{\mathrm{IW}}{ }^{16,17,21,28-30,33}$. However, the three parameters of $\mathrm{FE}_{\mathrm{IW}}$ 


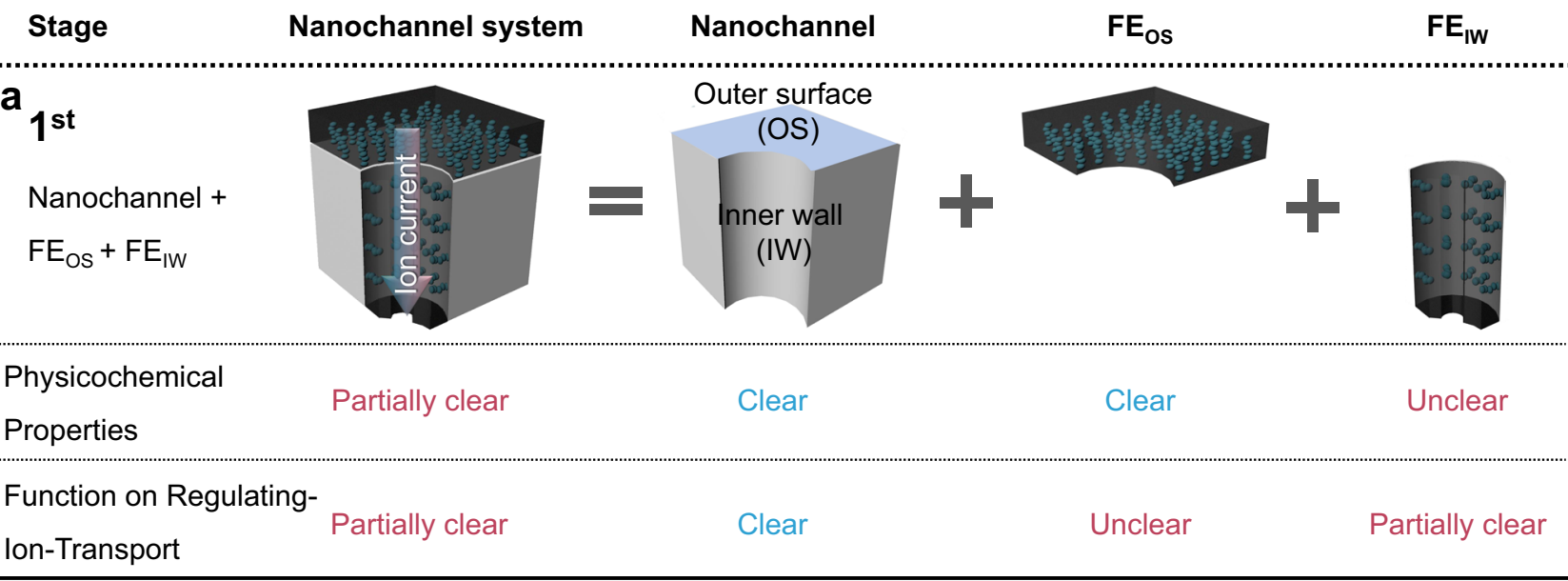

\section{b}

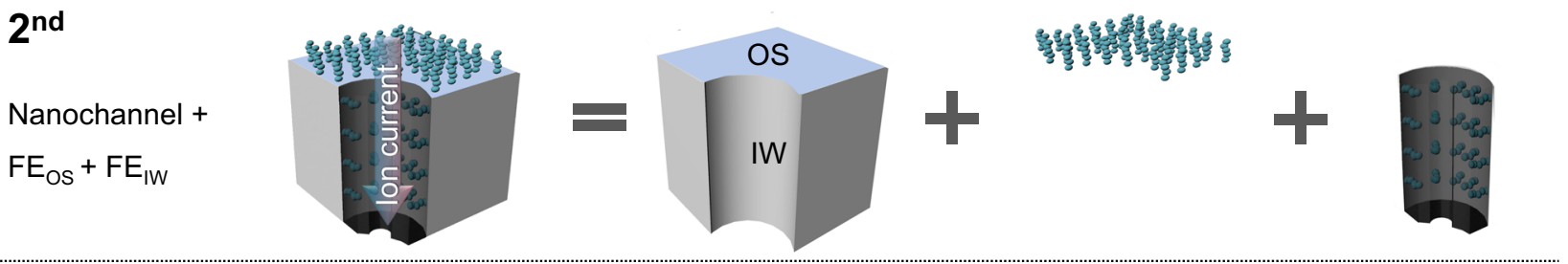

Physicochemical

Properties

Partially clear

Clear

Clear

Unclear

$\begin{array}{lll}\begin{array}{l}\text { Function on Regulating- } \\ \text { lon-Transport }\end{array} & \text { Partially clear } & \text { Clear }\end{array}$

\section{C}

\section{$3^{\text {rd }}$}

Nanochannel +

$\mathrm{FE}_{\mathrm{os}}$ (only)
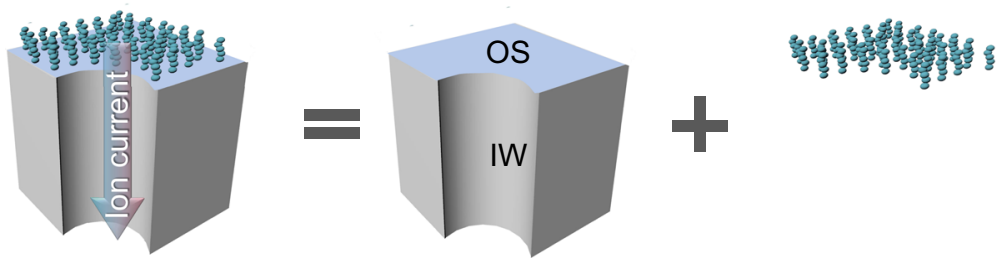

Physicochemical

Properties

Clear

Clear

Clear

Function on Regulating-

Ion-Transport

Clear

Clear

Clear

Two black shades refer to the two "black boxes" : one is the role of $\mathrm{FE}_{\mathrm{OS}}$ on regulating-ion-transports and the other is unclear physicochemical properties of $\mathrm{FE}_{\mathrm{IW}}$.

Fig. 1 Designed nanochannel-systems attached with $\mathbf{F E}_{\mathbf{O}}$ and $\mathbf{F E}_{\mathbf{I w}}$. a Stage 1, $\mathrm{FE}_{\mathrm{OS}}$ and $\mathrm{FE}_{\mathrm{IW}}$ immobilized as a whole, in which the role of $\mathrm{FE} \mathrm{OS}_{\mathrm{OS}}$ on ion transport and the properties of $\mathrm{FE}_{\mathrm{IW}}$ are inexplicit (two "black boxes" exist). b Stage 2, $\mathrm{FE}_{\mathrm{OS}}$ and $\mathrm{FE}_{\mathrm{IW}}$ as distinct part, in which the role of $\mathrm{FE}$ OS on ion transport began to be paid attention and investigated, but the properties of $\mathrm{FE}_{\mathrm{IW}}$ are still inexplicit (start to open the 1st "black box"). c Stage 3, in this work, independent $\mathrm{FE}_{\mathrm{OS}}$ without $\mathrm{FE}_{\mathrm{IW}}$ in nanochannel-system for regulating-ion-transport (further reveal the 1st "black box"), in which the properties of both $\mathrm{FE}_{\mathrm{OS}}$ and nanochannels are measurable, making the properties of the whole nanochannel-system explicit to a great extent (avoid the trouble from the 2 nd "black box").

used in equation were unmeasurable, as (1) the depth of FE at IW, (2) the surface charge, and (3) the diameter of the nanochannels after attaching $\mathrm{FE}_{\mathrm{IW}}$. Hence, the hypotheses of the above three parameters were generated in the 1st and 2nd stage, which is unavoidable: (1) FE may not completely cover IW, but leaving the blank area of the deep IW hard for FE to reach ${ }^{34}$. (2) The surface charge of $\mathrm{FE}_{\mathrm{IW}}$ is substituted by the measurable surface charge of $\mathrm{FE}_{\mathrm{OS}}$. Surface charge density is usually different between $\mathrm{FE}_{\mathrm{OS}}$ and $\mathrm{FE}_{\mathrm{IW}}$, due to their different grafting densities $^{22}$. Sometimes the charge of $\mathrm{FE}_{\mathrm{OS}}$ and $\mathrm{FE}_{\mathrm{IW}}$ inversed caused by the local polarization ${ }^{35}$. (3) The decrement of nanochannel diameter is roughly estimated by subtracting the straighten length of FE $\left(D-2 L_{\mathrm{SM}}\right.$, where $D$ represent the diameter of nanochannels and $L_{\mathrm{SM}}$ represent the straighten 

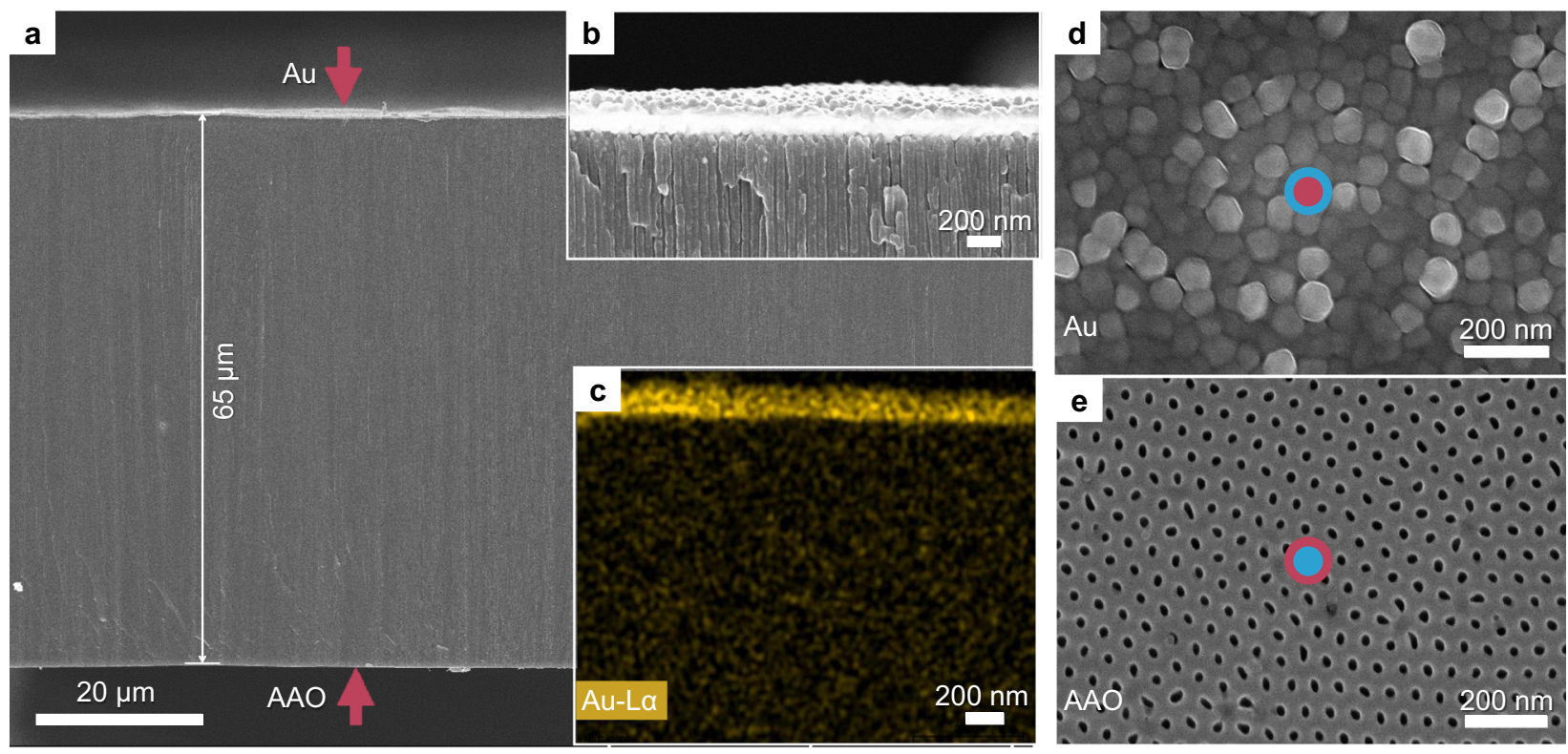

f Nanochannel System
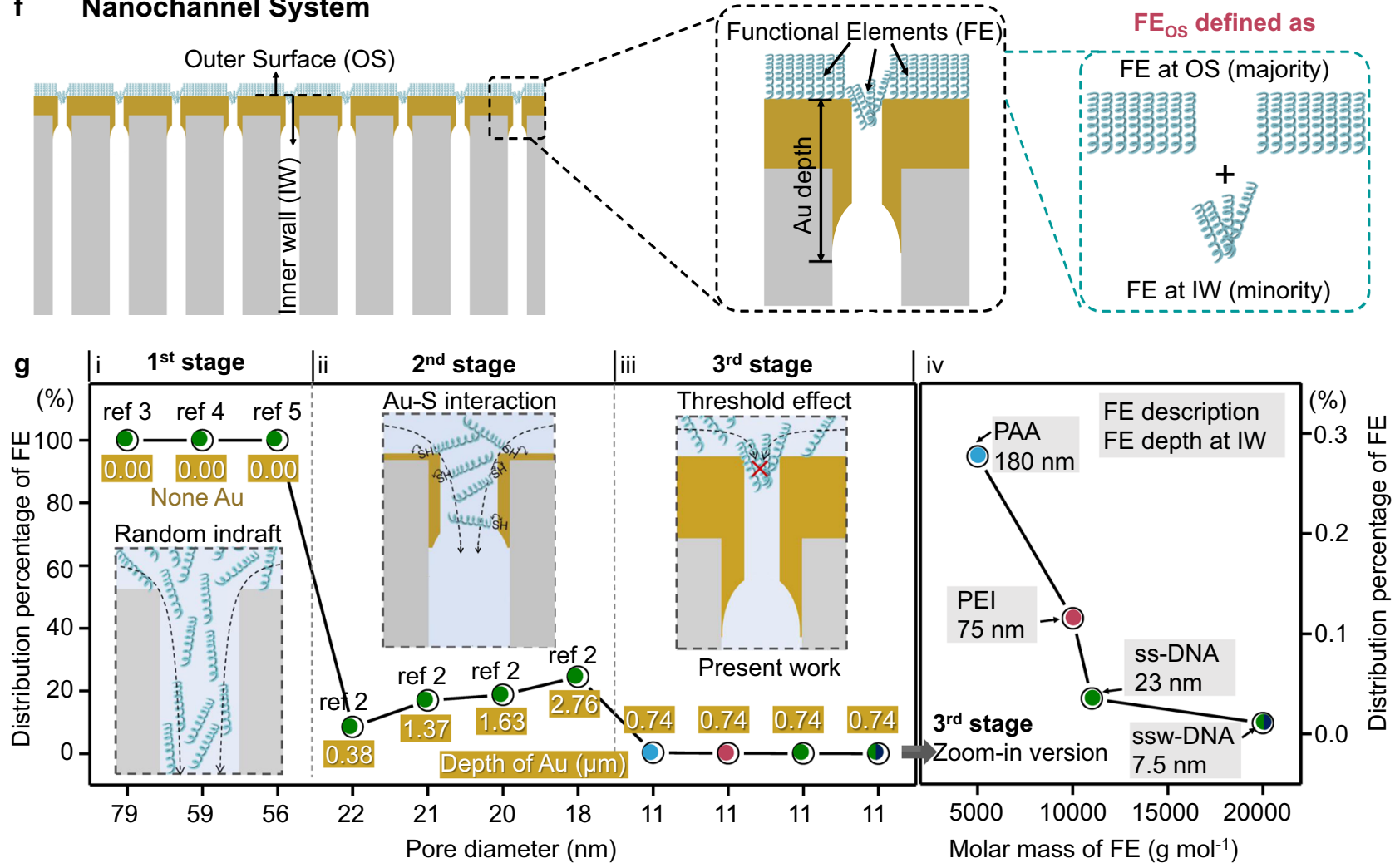

(1) Distribution percentage of FE $=\mathrm{FE}$ depth at IW $/$ total depth of IW, i.e. PAA percentage $=180 \mathrm{~nm} / 65 \mu \mathrm{m}=0.27 \%$

(2) $\bigcirc$ refer to PAA; $\bigcirc$ refer to PEl; $\bigcirc$ refer to ss-DNA, all ss-DNA are with same sequence; $\bigcirc$ refer to ssw-DNA, an double-stranded DNA amplified structure consisting of ss-DNA as a part

length of $\mathrm{FE})^{36}$. However, the $\mathrm{FE}$ are mostly not straight, i.e., single-strand $\mathrm{DNA}^{25}$. The results from the numerical simulations, therefore, deviate from experiments and even have randomness, which indicates that the above hypotheses bring the deviations or even sometimes errors. In our nanochannel-system, we measured (1) the depth of the part of $\mathrm{FE}_{\mathrm{OS}}$, (2) the surface charge, and (3) the diameter of nanochannels after attaching $\mathrm{FE}_{\mathrm{OS}}$ using ToFSIMS, SSZPA, and $\mathrm{AFM}^{37}$, respectively (Figs. $2 \mathrm{~g}, 3 \mathrm{~d}, 3 \mathrm{e}$ and Figs.
S11, S12). Both the qualitative and the quantitative variation of ICR behavior from numerical simulations fitted well with the experimental results, which indicated the explicit regulatingion-transport accomplished in 3rd stage nanochannel-system (Fig. 3f, g).

One of the important features of $\mathrm{FE}$ is of versatile physical and chemical properties, nanochannel-system adapt to a broad range of applications spanning from osmotic energy conversion 
Fig. 2 The characterization of none@OS and the FE $_{\text {os. }}$ a SEM image of none@OS from sectional view. The thickness of nanochannels is $65 \mu \mathrm{m}$. $\mathbf{b}$ Zoom-in version of Au coating side. c The corresponding energy dispersive X-ray spectroscopy of $\mathbf{b}$. $\mathbf{d}$, e SEM images of the OS coated with Au (d) and without Au (e) of none@OS from top view. f A scheme showing the present nanochannel-system and the FE distribution near the opening of nanochannel-system. The exposed surface to FE in none@OS includes OS and IW, of which the whole OS and a tiny fraction of IW are attached with FE herein. The FE consists of all $\mathrm{FE}_{\mathrm{OS}}$ and a very small amount of $\mathrm{FE}_{\mathrm{IW}} \cdot \mathbf{g}$ Comparison of the distribution percentage of $\mathrm{FE}$ at the IW in the (i) 1st, (ii) 2nd, and (iii) 3rd stage. In the 1st stage, the FE occupy the total depth of IW $(\approx 100 \%)$ through the random indraft of FE (the inset) $26,27,29$. In the 2 nd stage, the distribution percentage of FE at IW decrease down to $5-30 \%$ through the Au-S interaction between thiol-modified FE and IW (the inset) ${ }^{23}$. In the $3 r d$ stage, the distribution percentage of FE at IW sharply decline near zero. In the (iii) zoom-in version (iv), the distribution percentage of FE decrease with their molar mass, which demonstrates the threshold effects in the 3rd stage.

devices $^{15-17,33,38}$ to biosensors $26,27,39-41$. Meanwhile, a nanochannel system combining with electrochemistry in a confined space is now a crucial promising field ${ }^{42,43}$, which is utilized to dynamically monitor the single molecule ${ }^{44}$, understand the chemical reaction $^{45}$, characterize the single particle ${ }^{46}$, and probe single living cell ${ }^{47}$, etc. Here, we investigated whether the nanochannel-system in 3rd stage with $\mathrm{FE}_{\mathrm{OS}}$ explicit regulatingion-transport could fulfill the applications above or even with special performances to the 1 st and 2 nd.

Impact from $\mathrm{FE}_{\mathrm{O}}$ on osmotic energy conversion devices. The osmotic energy conversion devices were fabricated using nanochannels with only $\mathrm{FE}_{\mathrm{OS}}$ without $\mathrm{FE}_{\mathrm{IW}}$ (Fig. $4 \mathrm{a}-\mathrm{d}$ and Figs. S16-S19) ${ }^{15-17}$. The output power density of nanochannel system with independent $\mathrm{FE}_{\mathrm{OS}}$ was estimated according to the equation $P_{\mathrm{L}}=I^{2} / R_{\mathrm{L}}$, where $I$ is the current across the circuit and $R_{\mathrm{L}}$ is the external load resistance (Fig. S21). It was found that (1) output max power density increased with PAA concentration owing to the enhanced ion selectivity (Fig. $4 \mathrm{e}$ and Fig. S21); (2) $R_{\text {channel }}$ was almost unchanged with increase of concentration PAA (Fig. 4e and Fig. S21), where $R_{\text {channel }}$ was the internal resistance of the nanochannels. For (1), it is easy to understand the selectivity of the cell increased with the negative-charged $\mathrm{FE}_{\mathrm{OS}}(\mathrm{PAA})^{20}$. For (2), we speculated the $R_{\text {channel }}$ did not obviously change with the $\mathrm{FE}_{\mathrm{OS}}$. In order to verify our assumptions, two $\mathrm{FE}_{\mathrm{OS}}$ layer by layer (LbL) assembled on the OS of nanochannels (PAA and PEI sequentially in Fig. 4f-h and Figs. S22-S24; and PEI and PAA sequentially in Figs. S25 and S26) ${ }^{30,48}$. No distinctly increase of $R_{\text {channel }}$ was observed during two $\mathrm{FE}_{\mathrm{OS}}$ sequential assembly at the OS in the 3rd stage (Fig. 4i) owing to the ignored resistance induced by $\mathrm{FE}_{\mathrm{OS}}$, while $R_{\text {channel }}$ increased with the LbL assembly of PAA and PEI at the IW in the 1st and 2nd stages (Fig. S27). The above results demonstrated that the increase of $\mathrm{FE}_{\mathrm{OS}}$ (with selectivity) enhanced the "output max power density" of osmotic energy conversion devices (3rd stage), without obviously raising $R_{\text {channel }}$, which indicated a new conceptual route to design large power density nanochannel-system ${ }^{27,49-51}$.

Impact from $\mathrm{FE}_{\mathbf{O}}$ on biosensors. We further demonstrated a sensing strategy employing single-strand DNA probes as $\mathrm{FE}_{\mathrm{OS}}$ in the nanochannel-systems (3rd stage) (Fig. 5a), for the detection of a broad range of targets including inorganic ions $\left(\mathrm{Hg}^{2+}\right.$ with 1 pM limit of detection, LoD), small molecules (ATP with $1 \mathrm{pM}$ LoD), proteins (lysozyme with $1 \mathrm{pM}$ LoD), and cancer cells (MCF-7 cells with 400 cells $\mathrm{mL}^{-1}$ LoD) (Fig. 5b, c and Fig. S28). The targets were specifically captured by the designed $\mathrm{FE}_{\mathrm{OS}}$ as probe and tailored the surface charge of the OS locally, which affect the asymmetry of surface potential in between OS and IW and change the ion transport in form of $f_{\text {rec }}$ signal. To confirm the sensing mechanism above, we took ATP detection using sswDNA as an example. In ssw-DNA structure, one kind of repeating units in ssw-DNA was designed as ATP aptamer, which specifically bonded with ATP and caused the disassembly of ssw-DNA
(Fig. 5d-g and Fig. S29). The variation of surface potential under the disassembly of ssw-DNA triggered by different concentration ATP was quantitatively characterized through the electrochemical approaches (Fig. 5h and Fig. S30). The mechanism was also confirmed by the change grafting density of DNA at OS (Fig. S31). The selective detection for ATP was also realized based on surface-charge-response sensing mechanism (Fig. 5h, i). In the 1st and 2nd stage, because probes (as $\mathrm{FE}_{\mathrm{IW}}$ ) were immobilized at the IW, a confined space usually with diameter $<100 \mathrm{~nm}$, the targets with a size beyond the diameter of nanochannels can't sufficiently contact with probe and efficiently recognized. In the 3rd stage, the OS possess the receptive characteristic for probes or targets with the size beyond the diameter of nanochannels. The recognition between probes and targets took place at the OS, which is relatively more free-spaces compared with IW in nanochannels. Here, we realized a nearly "universal" biosensor approach according to the two successful sensing processes: one is the DNA amplifications (ssw-DNA) as the probes, whose diameter is larger than the diameter of the nanochannel-system (some probes is up to 30 -fold larger in Fig. 5e-g), and the other is the MCF-7 cells as the targets, whose diameters is about than 2 magnitudes larger than the diameters of the nanochannel-system.

\section{Discussion}

In conclusion, we have built a de novo designed "minimalist" nanochannel-system with an explicit regulating-ion-transport feature, which has been achieved using $\mathrm{FE}_{\mathrm{OS}}$ independently without $\mathrm{FE}_{\mathrm{IW}}$. The troubles from the "black box" for the properties of $\mathrm{FE}_{\mathrm{IW}}$ in the 1st and 2nd stage are well avoided without using $\mathrm{FE}_{\mathrm{IW}}$, meanwhile the other "black box", the role of $\mathrm{FE}_{\mathrm{OS}}$ on regulating-ion-transport, are further demonstrated from the "coadjutant" of $\mathrm{FE}_{\mathrm{IW}}$ in the 2nd stage to the "monodrama" in the 3rd stage. The use of independent $\mathrm{FE}_{\mathrm{OS}}$ is a new attempt to separate the enrichment, screening, and recognition process taking place at OS from the ion transport process taking place at IW within a confined space at the nanoscale. Furthermore, utilization of independent $\mathrm{FE}_{\mathrm{OS}}$ under a less restricted environment than $\mathrm{FE}_{\mathrm{IW}}$ will endow nanochannel-systems with (1) more measurable properties, such as hydrophobicity (the contact angle measurement is available for $\mathrm{FE}_{\mathrm{OS}}$, which is hardly achieved for $\mathrm{FE}_{\mathrm{IW}}$ in nanoscale confined space) (Fig. S32), (2) more characteristic technologies, such as X-ray photoelectron spectroscopy (XPS), ion microprobe mass analysis (IMMA) (the detection on $\mathrm{FE}_{\mathrm{IW}}$ requiring for destructive treatment, such as ion thinning, but not necessary for $\mathrm{FE}_{\mathrm{OS}}$ ), (3) more new performances limited to diffusions in nanochannels previously, such as reusage of FE (Fig. S33), rapid responses to targets (Fig. S34). The FE $\mathrm{OS}$ in 3rd stage like "ignition system" trigger the regulatable substrate transports through nanochannels and the abroad application space of nanochannel-systems.

\section{Methods}

Materials. Poly (acrylic acid) (PAA, MW 5000) was purchased from Ryon Biological Technology (Shanghai, China). Polyethyleneimine (PEI, MW 10,000) 
a
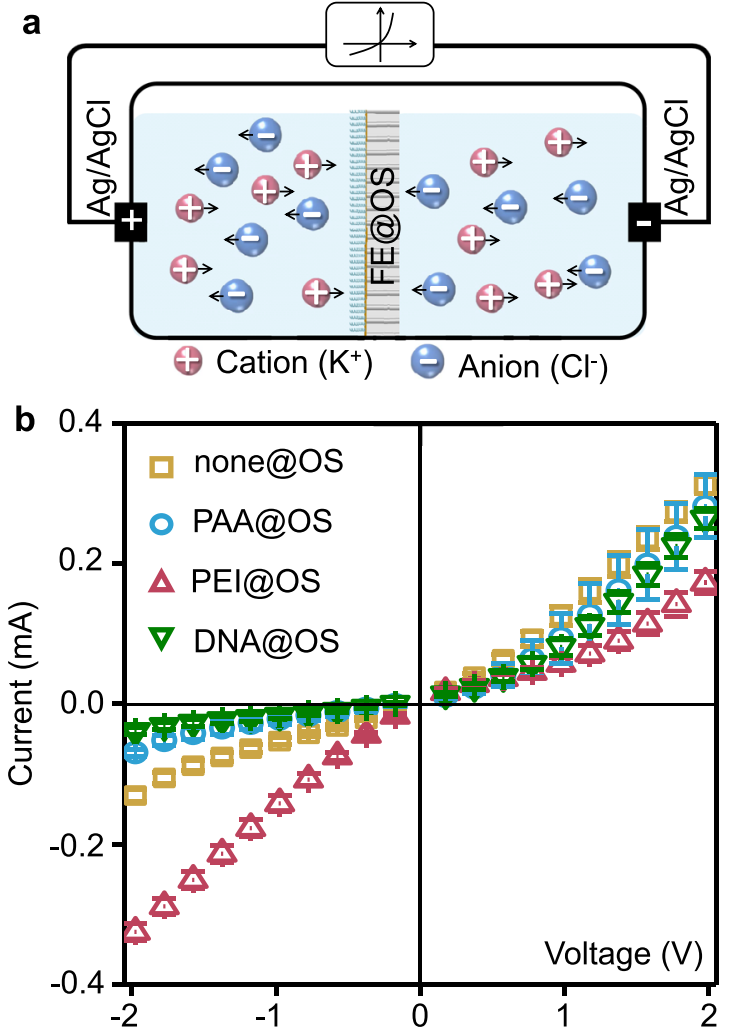

C

\section{From Poisson and Nernst-Planck equations}

$I=-\iint j_{i} d s=-\iint D_{i}\left(\nabla c_{i}+z_{i} c_{i} \frac{F}{R T} \nabla \varphi\right) d s$

$\nabla \varphi$ : charge ds : diameter and distribution

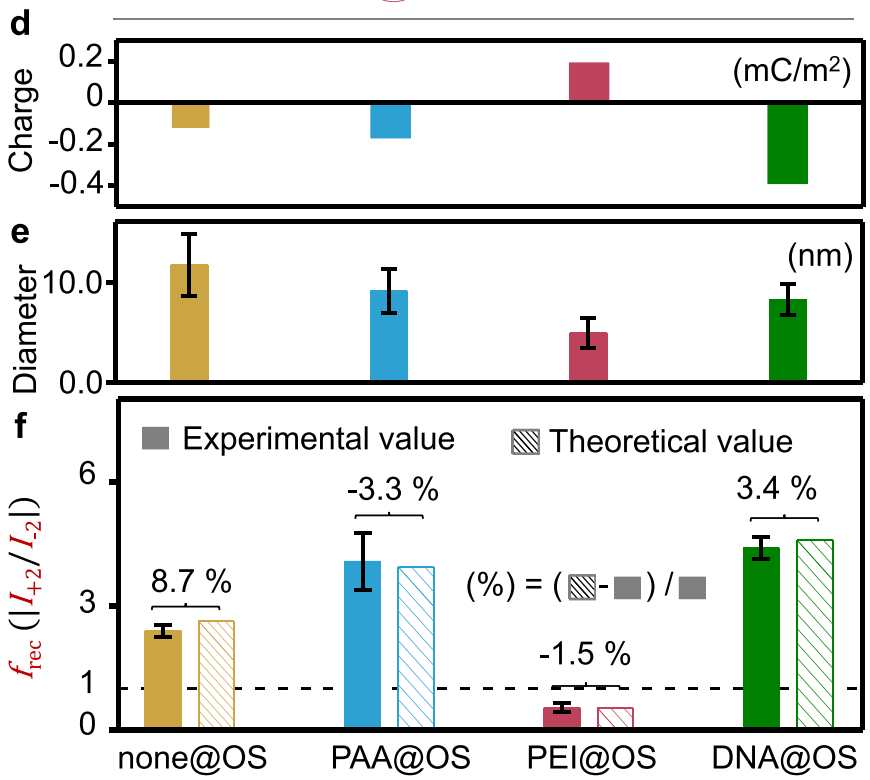

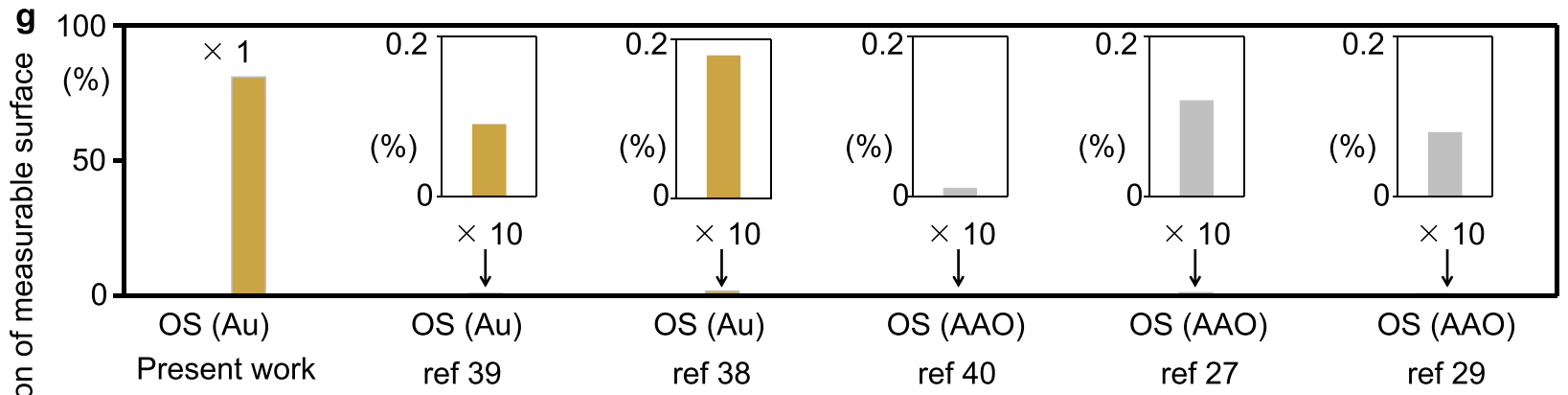

Fraction of measurable surface $=\mathrm{OS}$ area (all occupied by FE) / total area occupied by FE in nanochannel system

Fig. 3 Explicit role of $\mathbf{F E}_{\mathbf{o s}}$ in regulating-ion-transport. a Scheme of a two-electrode cell. $\mathbf{b} \mathrm{I}-\mathrm{V}$ curves characterizing the ion transport through nanochannel-system. c The PNP equation was used for numerical simulations of the effect from $\mathrm{FE}_{\mathrm{OS}}$ on the ion transport through nanochannel, where two variables remain: $\nabla c_{i}$ and $\nabla \varphi$ valued by the surface charge density $(\sigma)$ (measured by SSZPA, Fig. 3d) (1), the diameter of nanochannels (measured by AFM, Fig. 3e) (2) and the depth of FE at IW (measured by ToF-SIMS, Fig. 2g) (3) (More details in the section "Numerical simulation" in "Method" section). Thus, unless otherwise stated, the $I$ is available from numerical simulations using the three parameters (1), (2) and (3) measurable in the nanochannel-system with $\mathrm{FE}_{\mathrm{OS}}$ only but without $\mathrm{FE}_{\mathrm{IW}}$. $\mathbf{f}$ Comparison of the rectification ratio $\left(f_{\mathrm{rec}}\right)$ measured from I-V tests (experimental value) with the $f_{\text {rec }}$ from numerical simulations based on classical equations using measured parameters (theoretical value), but not estimated values like the previous works in 1st and 2nd stage. $\mathbf{g}$ Fraction of the measurable surface, which is the area proportion of the OS of measurable properties using mostly current test technologies, in the total surface occupied by FE (Details in Table S1). Data from the present work and the previous works using functional solid-state nanochannels $27,29,56-58$. For I-V tests, five chips were used to obtain each error bar. Statistics of diameters have been done by counting 100 nanochannels for each kind of nanochannels.

was purchased from Damas-beta. Tris (hydroxymethyl) aminomethane (Tris) was purchased from Alfa Aesar. $\mathrm{KCl}, \mathrm{NaCl}$, and $\mathrm{MgCl}_{2}$ were obtained from Aladdin reagent (Shanghai, China). Adenosine $5^{\prime}$-triphosphate (ATP) disodium salt solution, uridine Triphosphate (UTP), cytidine triphosphate (CTP) and guanosine triphosphate (GTP) were purchased from Sigma-Aldrich. HeLa and PC3 cells were obtained from Chinese Center for Typical Culture Collection (Wuhan, China) and cultured in DMEM (Gibco) supplemented with $10 \%$ (v/v) fetal bovine serum (FBS), $2 \mathrm{mg} / \mathrm{mL} \mathrm{NaHCO}_{3}$, and $100 \mathrm{U} / \mathrm{mL}$ antibiotics 15 (penicillin-streptomycin). MCF-7 cells were purchased from KeyGEN Biotech Co. Ltd. (Nanjing, China) and cultured in RPMI-1640 (Gibco) supplemented with $10 \% \mathrm{FBS}, 2 \mathrm{mg} / \mathrm{mL} \mathrm{NaHCO}$, and 100 $\mathrm{U} / \mathrm{mL}$ antibiotics (penicillin-streptomycin) at $37^{\circ} \mathrm{C}$ in a humidified $5 \% \mathrm{CO}_{2}$ atmosphere. AAO membranes were purchased from Pu-Yuan Nano Technology Co. Ltd. (Hefei, China). The thickness of AAO membrane is $65 \mu \mathrm{m}$. All solutions were prepared using Millipore Milli-Q water $(18 \mathrm{M} \Omega \mathrm{cm})$. All oligonucleotides are synthesized and purified by Sango Biotech Co. Ltd, (Shanghai, China). The sequences are shown in Tables S2 and S3.

Fabrication of nanochannel systems. Preparation of none@OS. A physical vapor deposition (PVD) method was employed using AE Nexdap PVD platform (Angstrom Engineering Inc.) to prepare nanochannels with independent $\mathrm{OS}^{22,23}$. Two kinds of depositing materials as $\mathrm{Au}$ and $\mathrm{Cr}$ were used. To enhance the stability of 


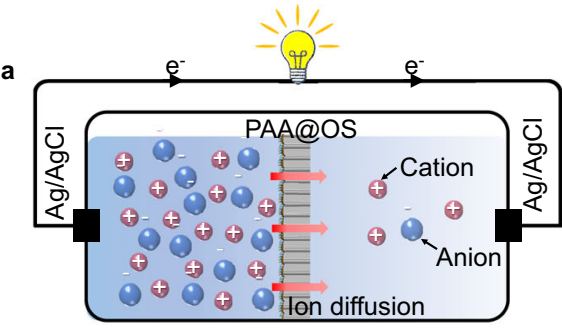

High concentrationLow concentration

c
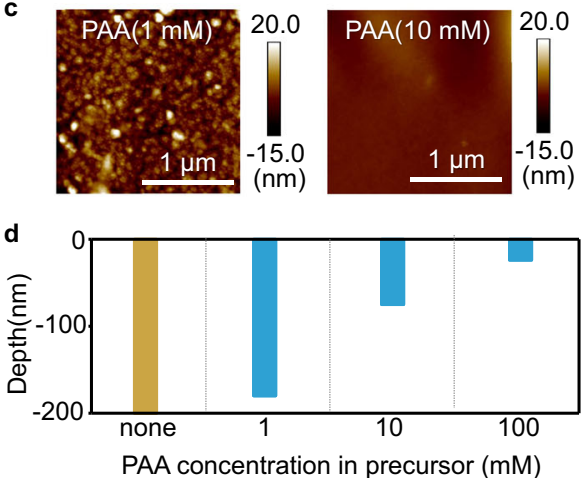

b 1 mM PAA

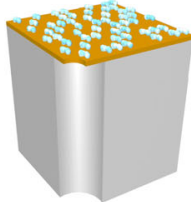

PAA@OS

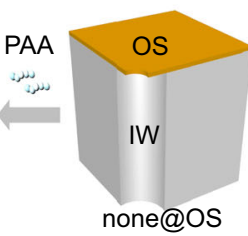

10 mM PAA

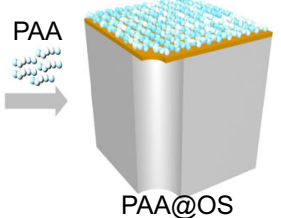

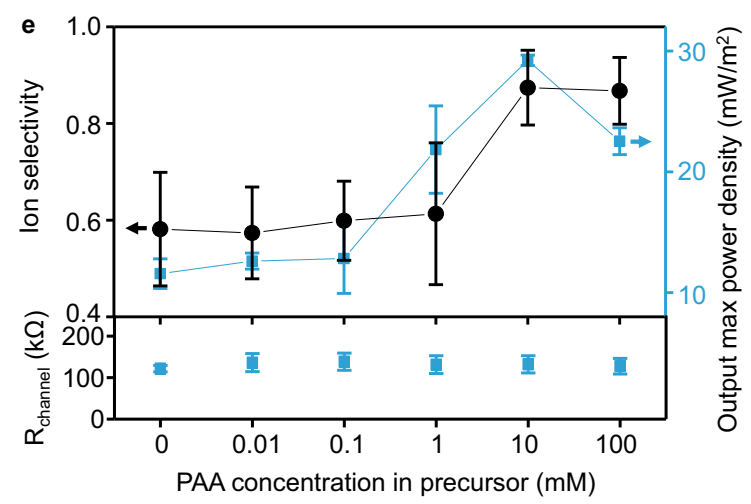

f
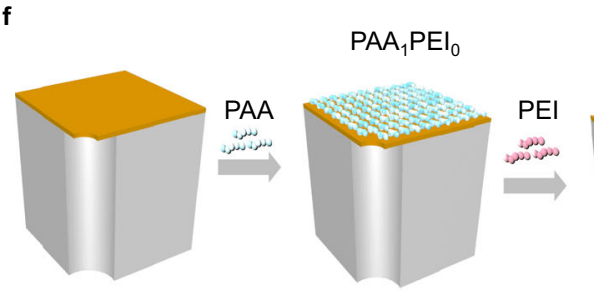

g
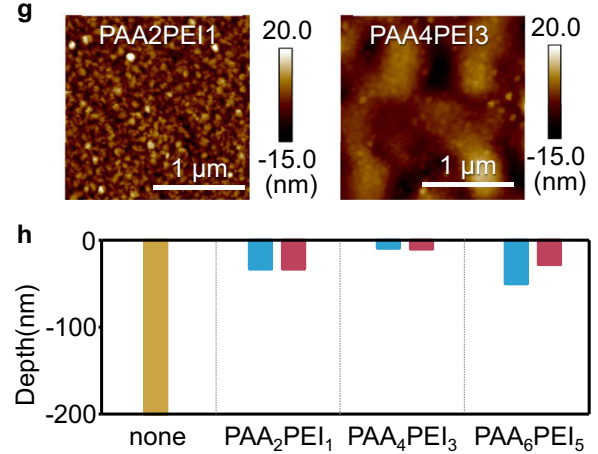

$\mathrm{PAA}_{1} \mathrm{PEI}_{1}$

$\mathrm{PAA}_{2} \mathrm{PEI}_{1}$
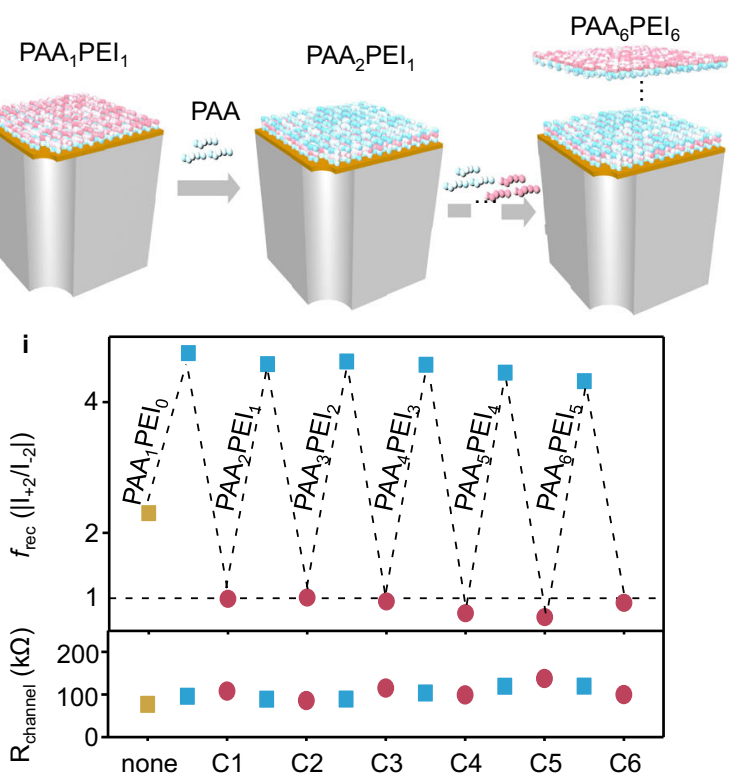

Fig. 4 Effect from $\mathbf{F E}_{\mathbf{o s}}$ on osmotic energy conversion devices. a A scheme showing the working mechanism of the osmotic energy conversion devices using nanochannel-system. The electricity by reverse electrodialysis is generated under salt gradient using a nanochannel system containing only $\mathrm{FE}_{\mathrm{OS}}$. b Fabrication of the nanochannel-system attached with PAA as FE (PAA@OS) using precursor with different PAA concentration (1 or 10 mM). c AFM of PAA@OS using 1 and 10 mM PAA in precursor. d Depth distribution of the part of FE $E_{O S}$ in nanochannel-system using precursor solution with different PAA concentration from ToF-SIMS. e Output max power density, ion selectivity and $R_{\text {channel }}$ of PAA@OS with different PAA concentration in precursor. $\mathbf{f}$ Scheme showing LbL assembly of PAA and PEI at OS sequentially. $\mathbf{g}$ AFM of the OS after LbL assembly. $\mathbf{h}$ Depth distribution of the part of FEOS (PAA and PEI) in nanochannel-system after LbL assembly. $\mathbf{i}$ Reversal $f_{\text {rec }}$ through the LbL assembly of PAA and PEl and corresponding $R_{\text {channel. }}$ The numerical simulations of two samples above, $\mathrm{PAA}_{4} \mathrm{PEI}_{3} @ \mathrm{OS}$ and $\mathrm{PAA}_{4} \mathrm{PEI}_{4} @ \mathrm{OS}$, using their measured parameters were performed, which well fit the experimental results (Fig. S24). For the osmotic energy conversion devices, five devices using FE@OS were assembled to obtain each error bar of power density and $R_{\text {channel. }}$

$\mathrm{Au}$, one layer of $\mathrm{Cr}$ with a thickness of $10 \mathrm{~nm}$ was first deposited at the one side of $\mathrm{AAO}$. The Au was followed deposited at the same side of $\mathrm{Cr}$ layer. The circular targets were approximately parallel to the AAO membranes, ensuring the deposition direction perpendicular to the membranes. The deposition was taken by the successive deposition without replacing the target materials or releasing vacuum. The successive deposition ensured no secondary pollution at the first deposited layer. The low depositing speed of Au was applied as $0.1 \mathrm{~nm} \mathrm{~s}^{-1}$ and the time was $2000 \mathrm{~s}$. The depositing speed was calibrated by the deposited thickness on the surface of the flat silicon wafer at nanometer level. The as-synthesized sample was named none@OS.
Preparation of PAA@OS,PEI@OS, and DNA@OS. Aqueous solution including PAA or PEI with different concentrations was prepared. Before attaching PAA or PEI, the Au-coated OS in none@OS was treated by Ar plasma for $60 \mathrm{~s}$ to remove impurities. Then, the PAA solution or the PEI solution was dip-coated at the OS. The dip-coating continued for $2 \mathrm{~min}$. Then the sample was washed throughout by water, and dried under $\mathrm{N}_{2}$ gas. The sample was named PAA@OS and PEI@OS, respectively. Thiol-modified DNA was attached at the OS of none@OS using similar dip-coating method. The reaction time continued for $60 \mathrm{~min}$ and the ample was named DNA@OS. The as-prepared samples were then applied for further measurement and characterizations. 
a

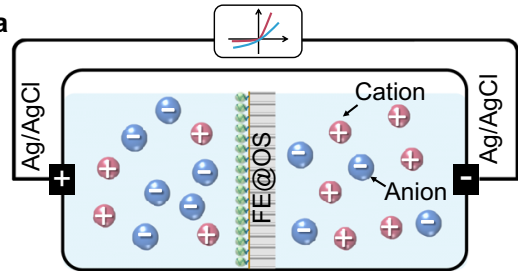

b DNA aptamer

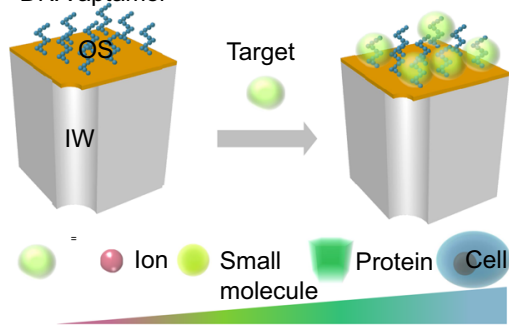

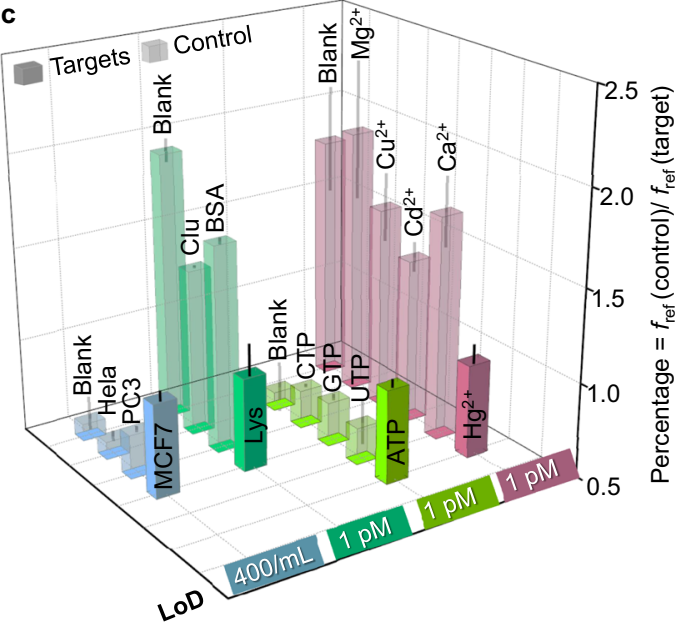
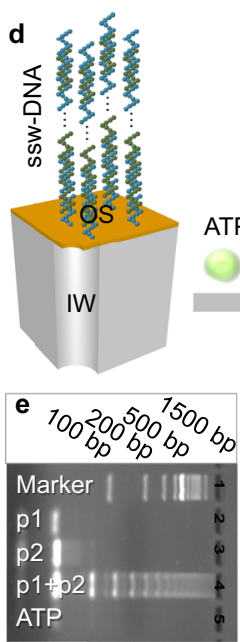
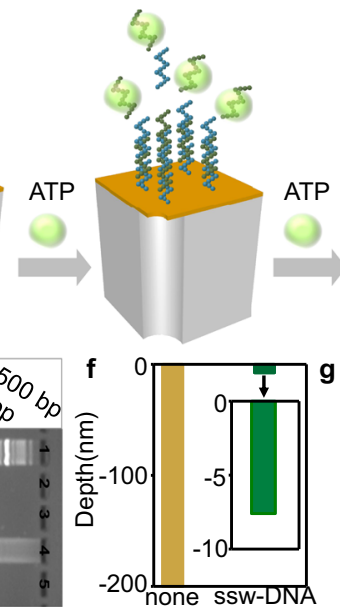
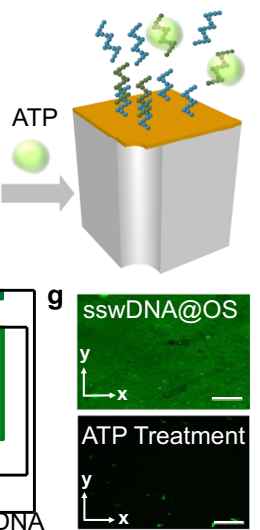
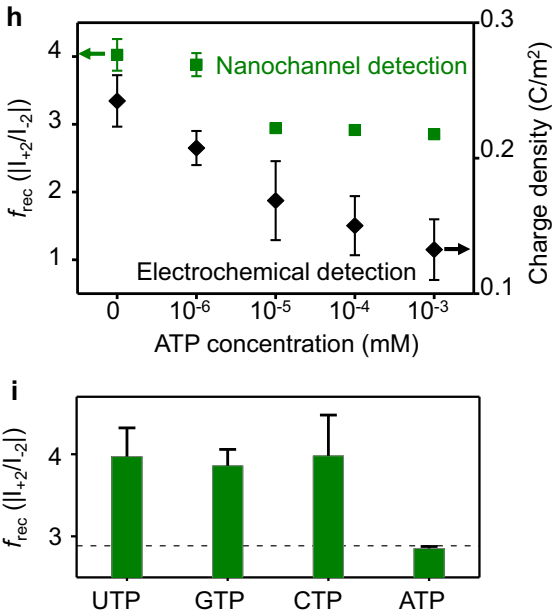

Fig. 5 Sensing performances of $\mathbf{F E}_{\text {os }}$ as probes. a A scheme showing the working mechanism of the sensor using FE $E_{O S}$ as probes. $\mathbf{b}$ Capture process of multi-scale targets through designed single-stand DNA. c Sensitivity and selectivity of the DNA@OS (DNA is a designed sequence specifically bonding with targets) for the recognition of ions $\left(\mathrm{Hg}^{2+}\right.$ ), small molecules (ATP), protein (Lysozyme) and cells (MCF7). The selective detection of multiscale targets using $\mathrm{FE}_{\mathrm{O}}$ was realized based on the change of $f_{\mathrm{rec}}$ signal output induced by the surface charge at outer surface. LoD is defined as the limitation of detection for the targets. d Formation of "supersandwich" DNA structure (ssw-DNA) with long concatamers through the successive hybridization of alternating DNA unit. And gradual disassembly of ssw-DNA based on interaction between ATP and the repeat DNA units through increasing ATP concentration. e Agarose gel electrophoresis characterizing of the ssw-DNA: 1) DNA marker; 2) p1; 3) p2 (ATP ampter); 4) p1 + p2; 5) target+p1 + p2. f Depth distribution of the "supersandwich" DNA in nanochannel-system using ToF-SIMS. $g$ Laser scanning confocal microscopy of the OS after the assembly (top) and the disassembly of ssw-DNA (bottom). The scale bar is $20 \mu \mathrm{m}$. $\mathbf{h}$ The LoD of ATP using ssw-DNA as probe based on nanochannel method and electrochemical method (Fig. S30). i Specificity of ssw-DNA@OS for ATP, in contrast with other NTPs. For the sensing performances part, five sensors using FE@OS were established to obtain each error bar of sensitivity and specificity.

LbL self-assembly of charged polyelectrolyte. For LbL self-assembly of PAA and PEI on the outer surface of AAO/Au nanochannels, the process was described as follows: an aqueous solution containing PAA $(1 \mathrm{mM})$ was firstly spread onto at the OS of none@OS for the adsorption of PAA polyelectrolyte, and the modification time was 2 min. After the adsorption of first layer of PAA, the solution of PEI was then spread on the same side of none@OS. Subsequently, other alternative layers of PAA/PEI were also electrostatically deposited on the OS of none@OS using the same procedure.

Assembly and disassembly of ssw-DNA. The DNA supersandwich structure (ssw-DNA) was prepared by mixing the thiol-modified cp-DNA solution $(1 \mu \mathrm{M})$ into the mixture of equimolar P1 and P2 $(1 \mu \mathrm{M})$ for $1 \mathrm{~h}$. Then the solution containing ssw-DNA was dropped onto the OS of none@OS, and the reaction time was $60 \mathrm{~min}$, named ssw-DNA@OS. The disassembly of ssw-DNA was achieved by treating the ssw-DNA@OS with different concentrations of ATP $(1 \mathrm{nM}-1 \mu \mathrm{M})$ for $60 \mathrm{~min}$.

Detection of $\mathbf{H g}^{2+}$, ATP, lysozyme, and MCF-7 cells. The aptamer solution $(1 \mu \mathrm{M})$ for different targets were dropped onto the OS of none@OS at room temperature for $60 \mathrm{~min}$. After that, 6 -mercapto-1-hexanol $(\mathrm{MCH}, 100 \mathrm{nM})$ was added onto the OS for $60 \mathrm{~min}$ to prevent nonspecific adsorption. Subsequently, the aptamer modified nanochannels was treatment by the suspension containing various targets. The treatment time was $60 \mathrm{~min}$. After the interaction, the sample was washed by buffer to remove the nonspecifically adsorbed targets. And then the as-treated samples were then applied for characterizations.

Cells were first cultured in flasks with Dubelcco's Modified Eagle's Medium (DMEM) at $37^{\circ} \mathrm{C}$ under $5 \% \mathrm{CO}_{2}$ in the cell incubator followed by centrifuging at $600 \times g$ for $5 \mathrm{~min}$, and re-dispersed in PBS $(10 \mathrm{mM}, \mathrm{pH} 7.4)$ with a density of $5 \times 10^{5}$ cells $\mathrm{mL}^{-1}$. A certain amount of cells suspension reacted with aptamer modified nanochannels for $60 \mathrm{~min}$. After cells capture, the sample was washed by PBS buffer to remove the nonspecifically adsorbed cells. And then the as-prepared membranes were then applied for characterizations.

Characterization. SEM images and EDX analyses were taken with a field-emission scanning electron microscope (SU8010, Hitachi, Japan) equipped with Energy Dispersive Xray spectroscopy (EDS, BRUKER AXS, Germany). All samples were coated with carbon $(5 \mathrm{~nm})$ prior to SEM examinations. Secondary ion mass spectra of as-prepared samples were characterized by ToF-SIMS V (IONTOF, GmbH). A Bi liquid metal primary ion source was applied with an angle of $45^{\circ}$ relative to the 
sample surface with a pulsed $\mathrm{Bi}^{3++}$ primary ion beam of $30 \mathrm{keV}$ and shave off fresh $60 \mu \mathrm{m} \times 60 \mu \mathrm{m}$ areas for each analysis. The ToF analyzer was installed at an angle of $90^{\circ}$ to the sample surface. Negative secondary ion spectra were collected. Mass calibration was carried out using standard procedures (mass resolving power $>5000$ ). The properties of OS of nanochannel system were characterized by an atomic force microscope (Multimode 8, Burke). The determination of OS's surface zeta potential of nanochannel system were carried out by using SSZPA (SurPASS, Anton Paar Ltd., Austria). The $\mathrm{pH}$ dependence of the zeta potential and the isoelectric point (IEP) were obtained in a $0.1 \mathrm{M}$ potassium chloride at the same electrical conductivity. The fluorescent measurement of nanochannel functionalized with fluorescent-dyed DNA at OS were performed by using laser scanning confocal microscope (LSM 880 confocal microscopy, Carl Zeiss) equipped with a FemtoSecond Laser (Coherent Inc.) The fluorescent-dyed membranes were placed at cover glass filling with water (around $1 \mathrm{~cm}^{2}$ membrane with $20 \mu \mathrm{L}$ water). For the analysis of assembly and dissembly process of ssw-DNA, the mixture was analyzed and observed using native PAGE gel. The gel was run in 16\% acrylamide (containing 19/1 acrylamide/bisacrylamide) solution with $1 \times$ TBE buffer, at $100 \mathrm{~V}$ constant voltage for $1.5 \mathrm{~h}$. The gel was directly imaged or stained with GelRed (Biotium) for $20 \mathrm{~min}$ to image the DNA position by using Tanon imaging system (Tanon 5200 Multi). The water contact angle measurements were operated on a DSA100 contact angle analyzer at ambient temperature and around 30\% humidity. A drop of water $(5.0 \mu \mathrm{L})$ was dropped onto the surfaces of nanochannel membranes.

The surface charge density for the OS with ssw-DNA grafting. The surface charge density with ssw-DNA grafting were tested using the system described in Fig. S28 and calculated based on the following equation ${ }^{52}$ :

$$
Q=\frac{2 n F A D_{0}^{1 / 2} C_{0}}{\pi^{1 / 2}} t^{1 / 2}+Q_{\mathrm{dl}}+n F A \Gamma_{0},
$$

where $n$ is electron transfer number, $\mathrm{F}$ is the Faraday constant, $A$ is the electrode area $\left(\mathrm{cm}^{2}\right), D_{0}$ is the diffusion coefficient $\left(\mathrm{cm}^{2} \mathrm{~s}^{-1}\right), C_{0}$ is the bulk concentration $\left(\mathrm{mol} \mathrm{cm}{ }^{-2}\right), Q_{\mathrm{dl}}$ is the capacitive charge $(C)$, and $\mathrm{n} F A \Gamma_{0}$ is the charge from the reduction of $\Gamma_{0}\left(\mathrm{~mol} \mathrm{~cm}^{-2}\right)$ of adsorbed redox marker.

The samples were mounted in between the two halves of a homemade electrochemical cell, which contains $1.0 \mathrm{~mL} \mathrm{KCl}$ solution in each cell. I-V plots were recorded by an electrochemical workstation (CHI, ShangHai) and Keithley 6487 picoammeter (Keithley Instruments, Cleveland, $\mathrm{OH}$ ). Two $\mathrm{Ag} / \mathrm{AgCl}$ electrodes were used to apply the potential. The working electrode was placed on the $\mathrm{Au}$ side and the reference electrode on the AAO side if not particularly mentioned. The effective area of the membrane for ion conduction test is about 7 $\mathrm{mm}^{2}$. All measurements were performed at room temperature, and each test was repeated for five times. Five membranes at least were used to obtain average values. To study the ionic rectification properties, a scanning voltage from -2.0 to $2.0 \mathrm{~V}$ was applied across the membrane. The ion concentration on the Au side and the $\mathrm{AAO}$ side was kept equal $(0.1 \mathrm{M} \mathrm{KCl}, \mathrm{pH} 7.0)$, if not specially mentioned. To study the energy conversion properties ${ }^{53}$ a salt gradient was designed, where the concentration of the solution on the Au side is higher than that on the AAO side if not particularly mentioned.

Numerical simulations. The ionic rectification phenomenon was theoretically investigated using a commercial finite-element software package COMSOL Multiphysics ${ }^{54,55}$.

$$
\begin{gathered}
J_{\mathrm{i}}=D_{\mathrm{i}}\left(\nabla c_{\mathrm{i}}+\frac{z_{\mathrm{i}} F c_{\mathrm{i}}}{R T} \nabla \varphi\right), \\
\nabla^{2} \varphi=-\frac{F}{\varepsilon} \sum z_{\mathrm{i}} c_{\mathrm{i}}, \\
\nabla \cdot J_{\mathrm{i}}=0 .
\end{gathered}
$$

Here, Eq. (2) is the Nernst-Planck equation that descripts the transport properties of a charge nanochannel and Eq. (3) is the Poisson equation that descripts the relationship between the electric potential and ion concentration in the nanochannels. The model is generally simplified using steady-state conditions (Eq. (4)). The electroosmotic flow was neglected in this work. The coupled Eqs. (2-4) can be solved utilizing appropriate boundary conditions (Eqs. 4, 6 and Table S4). The solution yields the concentration field $c_{\mathrm{i}}$ for all species and the potential $\varphi$ distributions in the nanochannels.

$$
\begin{gathered}
\mathbf{n} \cdot \nabla \varphi=-\frac{\sigma}{\varepsilon}, \\
\mathbf{n} \cdot J_{\mathrm{i}}=0 .
\end{gathered}
$$

The total ionic current through the nanochannel can be calculated:

$$
I=\iint J_{\mathrm{i}} \mathrm{d} s=-\iint D\left(\nabla c_{\mathrm{i}}+z_{\mathrm{i}} c_{\mathrm{i}} \frac{F}{R T} \nabla \varphi\right) \mathrm{d} s .
$$

The formula above was used for numerical simulations of the effect from $\mathrm{FE}_{\mathrm{OS}}$ on the ion transport through nanochannel, where $i$ refer to cation $\left(\mathrm{K}^{+}\right)$and anion $\left(\mathrm{Cl}^{-}\right)$and $J_{\mathrm{i}}$ refers to ionic flux ${ }^{16}$. When the properties of electrolyte are set, the invariants include: $D_{\mathrm{i}}$ as the diffusion coefficient, $c_{\mathrm{i}}$ as the concentration of $\mathrm{K}^{+}$and
$\mathrm{Cl}^{-}, z_{\mathrm{i}}$ as the valence of $\mathrm{K}^{+}$and $\mathrm{Cl}^{-}, T$ as the thermodynamic temperature. Meanwhile, in this formula, the constants include: $F$ as the Faraday's constant and $R$ as the ideal gas constant. As a result, two variables remain: $\nabla c_{\mathrm{i}}$ describes the concentration gradient of ion along nanochannels under external electric field and $\nabla \varphi$ describes the electrical potential gradient along nanochannels, in which the $\varphi$ is valued by the surface charge density $(\sigma)$ of the $\mathrm{FE}_{\mathrm{OS}}$ and the nanochannels (measured by SSZPA, Fig. 3d) (1). The $\nabla c_{\mathrm{i}}$ and the $\nabla \varphi$ are calculated by integrating the $c_{\mathrm{i}}$ of electrolyte and $\varphi$ over the cross-section area $(\mathrm{d} s)$. The integration of $\mathrm{d} s$ is valued by the diameter of nanochannels (measured by AFM, Fig. 3e) (2), and the depth of FE at IW (measured by ToF-SIMS, Fig. 2g) (3).

\section{Data availability}

The data that support the plots within this paper and other finding of this study are available from the corresponding author upon reasonable request.

\section{Received: 5 June 2020; Accepted: 13 January 2021;}

Published online: 10 March 2021

\section{References}

1. Kowalczyk, S. W., Blosser, T. R. \& Dekker, C. Biomimetic nanopores: learning from and about nature. Trends Biotechnol. 29, 607-614 (2011).

2. Bocquet, L. Nanofluidics coming of age. Nat. Mater. 19, 254-256 (2020).

3. Martin, C. R. \& Siwy, Z. S. Learning nature's way: biosensing with synthetic nanopores. Science 317, 331-332 (2007).

4. Keyser, U. F. Enhancing nanopore sensing with DNA nanotechnology. Nat. Nanotechnol. 11, 106-108 (2016).

5. Liu, L., Yang, C., Zhao, K., Li, J. \& Wu, H. Ultrashort single-walled carbon nanotubes in a lipid bilayer as a new nanopore sensor. Nat. Commun. 4, 2989 (2013).

6. Burns, J. R., Seifert, A., Fertig, N. \& Howorka, S. A biomimetic DNA-based channel for the ligand-controlled transport of charged molecular cargo across a biological membrane. Nat. Nanotechnol. 11, 152-156 (2016).

7. Ying, Y. L. et al. Manipulating and visualizing the dynamic aggregationinduced emission within a confined quartz nanopore. Nat. Commun. 9, 3657 (2018)

8. Liang, B. et al. Microporous membranes comprising conjugated polymers with rigid backbones enable ultrafast organic-solvent nanofiltration. Nat. Chem. 10, 961-967 (2018)

9. Hou, X., Hu, Y., Grinthal, A., Khan, M. \& Aizenberg, J. Liquid-based gating mechanism with tunable multiphase selectivity and antifouling behaviour. Nature 519, 70-73 (2015)

10. Lozada-Hidalgo, M. et al. Sieving hydrogen isotopes through two-dimensional crystals. Science 351, 68-70 (2016).

11. Lu, J. et al. Efficient metal ion sieving in rectifying subnanochannels enabled by metal-organic frameworks. Nat. Mater. https://doi.org/10.1038/s41563020-0634-7 (2020)

12. Xie, Q. et al. Fast water transport in graphene nanofluidic channels. Nat. Nanotechnol. 13, 238-245 (2018).

13. Simon, P., Gogotsi, Y. \& Dunn, B. Where do batteries end and supercapacitors begin? Science 6176, 1210-1211 (2014).

14. Liu, C. et al. An all-in-one nanopore battery array. Nat. Nanotechnol. $\mathbf{9}$, 1031-1039 (2014)

15. Siria, A. et al. Giant osmotic energy conversion measured in a single transmembrane boron nitride nanotube. Nature 494, 455-458 (2013).

16. Feng, J. et al. Single-layer $\mathrm{MoS}_{2}$ nanopores as nanopower generators. Nature 536, 197-200 (2016).

17. Gao, J. et al. High-performance ionic diode membrane for salinity gradient power generation. J. Am. Chem. Soc. 136, 12265-12272 (2014).

18. Zhang, Z. et al. Engineering smart nanofluidic systems for artificial ion channels and ion pumps: from single-pore to multichannel membranes. $A d v$. Mater. 32, 1904351 (2019).

19. Savariar, E. N., Thayumanavan, S. \& Krishnamoorthy, K. Molecular discrimination inside polymer nanotubules. Nat. Nanotechnol. 3, 112-117 (2008).

20. Tagliazucchi, M., Rabin, Y. \& Szleifer, I. Ion transport and molecular organization are coupled in polyelectrolyte-modified nanopores. J. Am. Chem. Soc. 133, 17753-17763 (2011).

21. Cao, L. et al. On the origin of ion selectivity in ultrathin nanopores: insights for membrane-scale osmotic energy conversion. Adv. Funct. Mater. 28, 1804189 (2018)

22. Li, X. et al. Role of outer surface probes for regulating ion gating of nanochannels. Nat. Commun. 9, 40 (2018).

23. Gao, P. et al. Distinct functional elements for outer-surface anti-interference and inner-wall ion gating of nanochannels. Nat. Commun. 9, 4557 (2018).

24. Acar, E. T., Buchsbaum, S. F., Combs, C., Fornasiero, F. \& Siwy, Z. S. Biomimetic potassium-selective nanopores. Sci. Adv. 5, v2568 (2019). 
25. Buchsbaum, S. F., Nguyen, G., Howorka, S. \& Siwy, Z. S. DNA-modified polymer pores allow PH- and voltage-gated control of channel flux. J. Am. Chem. Soc. 136, 9902-9905 (2014).

26. Liu, N. et al. Two-way nanopore sensing of sequence-specific oligonucleotides and small-molecule targets in complex matrices using integrated DNA supersandwich structures. Angew. Chem. Int. Ed. 52, 2007-2011 (2013).

27. Jiang, Y., Liu, N., Guo, W., Xia, F. \& Jiang, L. Highly-efficient gating of solidstate nanochannels by DNA supersandwich structure containing ATP aptamers: a nanofluidic implication logic device. J. Am. Chem. Soc. 134, 15395-15401 (2012).

28. Tagliazucchi, M., Rabin, Y. \& Szleifer, I. Transport rectification in nanopores with outer membranes modified with surface charges and polyelectrolytes. ACS Nano 7, 9085-9097 (2013).

29. Jiang, Y. et al. On the origin of ionic rectification in DNA-stuffed nanopores: the breaking and retrieving symmetry. J. Am. Chem. Soc. 139, 18739-18746 (2017).

30. Ali, M. et al. Layer-by-layer assembly of polyelectrolytes into ionic current rectifying solid-state nanopores: insights from theory and experiment. J. Am. Chem. Soc. 132, 8338-8348 (2010).

31. Siwy, Z. S. Ion-current rectification in nanopores and nanotubes with broken symmetry. Adv. Funct. Mater. 16, 735-746 (2006).

32. Sparreboom, W., van den Berg, A. \& Eijkel, J. C. T. Principles and applications of nanofluidic transport. Nat. Nanotechnol. 4, 713-720 (2009).

33. Lin, C., Combs, C., Su, Y., Yeh, L. \& Siwy, Z. S. Rectification of concentration polarization in mesopores leads to high conductance ionic diodes and high performance osmotic power. J. Am. Chem. Soc. 141, 3691-3698 (2019)

34. Xu, X. et al. Coordination of the electrical and optical signals revealing nanochannels with an 'onion-like' gating mechanism and its sensing application. NPG Asia Mater. 8, e234 (2016).

35. Lin, K., Lin, C. Y., Polster, J. W., Chen, Y. \& Siwy, Z. S. Charge inversion and calcium gating in mixtures of ions in nanopores. J. Am. Chem. Soc. 142, 2925-2934 (2020).

36. Huang, K. \& Szleifer, I. Design of multifunctional nanogate in response to multiple external stimuli using amphiphilic diblock copolymer. J. Am. Chem. Soc. 139, 6422-6430 (2017).

37. Yang, Q. et al. Array atomic force microscopy for real-time multiparametric analysis. Proc. Natl Acad. Sci. USA 116, 5872-5877 (2019).

38. Xiao, K., Jiang, L. \& Antonietti, M. Ion transport in nanofluidic devices for energy harvesting. Joule 3, 2364-2380 (2019).

39. Wang, C. et al. Dendrimer-Au nanoparticle network covered alumina membrane for ion rectification and enhanced bioanalysis. Nano Lett. 20, 1846-1854 (2020).

40. Wang, Y., Yang, Q., Zhao, M., Wu, J. \& Su, B. Silica-nanochannel-based interferometric sensor for selective detection of polar and aromatic volatile organic compounds. Anal. Chem. 90, 10780-10785 (2018).

41. Sun, Y. et al. A highly selective and recyclable NO-responsive nanochannel based on a spiroring opening-closing reaction strategy. Nat. Commun. 10, 1-8 (2019).

42. Lu, S., Peng, Y., Ying, Y. \& Long, Y. Electrochemical sensing at a confined space. Anal. Chem. 92, 5621-5644 (2020).

43. Ying, Y., Gao, R., Hu, Y. \& Long, Y. Electrochemical confinement effects for innovating new nanopore sensing mechanisms. Small Methods 2, 1700390 (2018).

44. Qing, Y., Tamagaki-Asahina, H., Ionescu, S., Liu, M. \& Bayley, H. Catalytic site-selective substrate processing within a tubular nanoreactor. Nat. Nanotechnol. 14, 1135-1142 (2019).

45. Cao, J. et al. Giant single molecule chemistry events observed from a tetrachloroaurate(III) embedded Mycobacterium smegmatis porin A nanopore. Nat. Commun. 10, 5668 (2019).

46. Gao, R. et al. A $30 \mathrm{~nm}$ nanopore electrode: facile fabrication and direct insights into the intrinsic feature of single nanoparticle collisions. Angew. Chem. Int. Ed. 57, 1011-1015 (2018).

47. Nadappuram, B. et al. Nanoscale tweezers for single-cell biopsies. Nat. Nanotechnol. 14, 80-88 (2019).

48. Mariani, S. et al. Layer-by-layer biofunctionalization of nanostructured porous silicon for high-sensitivity and high-selectivity label-free affinity biosensing. Nat. Commun. 9, 5256 (2018).

49. Kowalczyk, S. W. et al. Single-molecule transport across an individual biomimetic nuclear pore complex. Nat. Nanotechnol. 6, 433-438 (2011).

50. Probstein, R. F. Physicochemical Hydrodynamics Ch. 6 (Wiley, 2005).

51. Gao, J. et al. Understanding the giant gap between single-pore- and membrane-based nanofluidic osmotic power generators. Small 15, 1804279 (2019).
52. Steel, A. B., Herne, T. M. \& Tarlov, M. J. Electrochemical quantitation of DNA immobilized on gold. Anal. Chem. 70, 4670-4677 (1998).

53. Kim, D., Duan, C., Chen, Y. \& Majumdar, A. Power generation from concentration gradient by reverse electrodialysis in ion-selective nanochannels. Microfluid. Nanofluid. 9, 1215-1224 (2010).

54. White, H. S. \& Bund, A. Ion current rectification at nanopores in glass membranes. Langmuir 24, 2212-2218 (2008).

55. Lan, W. J., Holden, D. A. \& White, H. S. Pressure-dependent ion current rectification in conical-shaped glass nanopores. J. Am. Chem. Soc. 133, 13300-13303 (2011).

56. Wang, X. \& Smirnov, S. Label free DNA sensor based on surface charge modulated ionic conductance. ACS Nano 3, 1004-1010 (2009).

57. Lee, S. B. Antibody-based bio-nanotube membranes for enantiomeric drug separations. Science 296, 2198-2200 (2002).

58. Zhou, Y. et al. High-temperature gating of solid-state nanopores with thermoresponsive macromolecular nanoactuators in ionic liquids. Adv. Mater. 24, 962-967 (2012).

\section{Acknowledgements}

This work is supported by the National Natural Science Foundation of China (22090050 21974126, 21874121, and 51803194). This research is supported by the Hubei Provincial Natural Science Foundation of China (2020CFA037), Zhejiang Provincial Natural Science Foundation of China under Grant No. LY19B030001 and LD21B050001. The project is supported by the Open-end Funds from Engineering Research Center of NanoGeomaterials of Ministry of Education (NGM2019KF013) and the Fundamental Research Funds for National Universities, China University of Geosciences (Wuhan). This research work was supported by the Open Funds of the State Key Laboratory of Electroanalytical Chemistry (SKLEAC202003) and the National Key Research and Development Program of China (2018YFE0206900).

\section{Author contributions}

F.X. directed the project. P.G. conceived and designed the experiments. Q.M. fabricated devices, performed measurements and carried out data analysis with help from Y.L., R.W., H.X., and Q. D. carried out the numerical simulations. P.G., Q.M., and F.X. wrote the manuscript. All authors contributed to discussions.

\section{Competing interests}

The authors declare no competing interests.

\section{Additional information}

Supplementary information The online version contains supplementary material available at https://doi.org/10.1038/s41467-021-21507-7.

Correspondence and requests for materials should be addressed to P.G. or F.X.

Peer review information Nature Communications thanks Kai Huang and other, anonymous, reviewers for their contributions to the peer review of this work. Peer review reports are available.

Reprints and permission information is available at http://www.nature.com/reprints

Publisher's note Springer Nature remains neutral with regard to jurisdictional claims in published maps and institutional affiliations.

Open Access This article is licensed under a Creative Commons Attribution 4.0 International License, which permits use, sharing, adaptation, distribution and reproduction in any medium or format, as long as you give appropriate credit to the original author(s) and the source, provide a link to the Creative Commons license, and indicate if changes were made. The images or other third party material in this article are included in the article's Creative Commons license, unless indicated otherwise in a credit line to the material. If material is not included in the article's Creative Commons license and your intended use is not permitted by statutory regulation or exceeds the permitted use, you will need to obtain permission directly from the copyright holder. To view a copy of this license, visit http://creativecommons.org/ licenses/by/4.0/.

(C) The Author(s) 2021 